\title{
Strength building, data analytics and privacy: An urgent need to update the balance
}

\author{
Brian Rector*
}

At first glance the purpose and description of the new editorial section, Strengths, for community safety and wellbeing, look rather straight forward and non-controversial: "Working together with individuals, families, communities, and cultures to improve life quality, to build strength-based capacities, and to achieve social outcomes through innovative, data-driven analysis and measurable outcomes." I wish life were so simple.

Sometimes, innovation and data-driven analysis will take us to uncomfortable places. We are now aware of many examples where demonstrated results through analysis have challenged and even conflicted with our prior thinking and belief systems. What we may not recognize is that those individuals who at one time believed the world to be flat, may well be us today in the field of community safety.

As the Editor-in-Chief referenced in his editorial (Taylor, 2018), we collaborated with the Canadian Association of Chiefs of Police to hold a conference last April on advanced analytics and community safety. For me, the unofficial title of that conference was "not hot spotting". We focused upstream, tapping into emerging research that uses advanced techniques in combination with multiple data sources to improve safety and well-being outcomes for individuals. I believe the Journal can play a significant role in advancing this approach.

Advanced predictive analytics, deep learning, and artificial intelligence are all terms many will have heard, but applying this wide array of methodologies will require new kinds of collaboration, and at times completely new thinking if measurable outcomes in community safety are to be successfully achieved.

By new kinds of collaboration, I refer to public sector community safety and well-being service agencies striking formal collaboration agreements with advanced experts in mathematics and computer science. Collaboration may at times involve private companies that have invested in these advanced skill sets, and I would also highlight the potential in formal collaborations with experts and scholars working at universities in any of the cross-cutting thematic areas referenced throughout this Journal. Although the skill sets may be similar among private and not-for-profit researchers and analysts, just how open these analytics can be may differ under differing arrangements. For transparency and scrutiny, this is an important consideration, and an issue that must be specifically addressed in the framing documents that define any such collaboration.

Anytime we examine multiple data sets, especially data at the individual level, the important question of privacy must be addressed, and the balance between privacy and public safety must be carefully understood and applied. But how do we define that balance? Striking a balance between privacy and public safety is a phrase often used, but do we really know what it means in practical terms? Do we balance only when we discover that public safety has been so compromised that a change is required? Or do we seek and establish that balance proactively and on agreed-to criteria? I think we are currently more in the former stage. Unfortunately, what this sadly and too often means is, how many children and adults must die before we adjust? Criminal groups embrace technology. To not apply the best of our technologies to address the challenging issues that undermine CSWB collaborations may result in our defined measures of public safety deteriorating over time. A holding pattern is not good enough.

In some ways, the application of advanced analytics reminds me of those early advances in genetic research that leapt ahead of then-current legislation and created new ethical dilemmas. With time, legislation did adapt and new ethical standards were defined. Today, how the Journal of CSWB contributes to the research and discussions needed to similarly refine these checks and balances in public safety is an important and urgent role, and a role in which I am pleased to play some part.

\section{CONFLICT OF INTEREST DISCLOSURES}

The author declares there are no conflicts of interest.

\section{AUTHOR AFFILIATIONS}

* Research and Evidence Based Excellence, Saskatchewan Ministry of Justice and Ministry of Social Services, Regina, SK, Canada.

\section{REFERENCES}

Taylor, N. (2018). CSWB: Where complexity serves simple ideals [editorial]. Journal of Community Safety \& Well-being, 3(1). 